\title{
REVIEW
}

\section{Functionalized polymer nanofibers: a versatile platform for manipulating light at the nanoscale}

\author{
Pan Wang, Yipei Wang and Limin Tong
}

As an organic optical fiber with a diameter comparable to or less than the wavelength of light, polymer nanofibers have been attracting increasing attention as a platform for manipulating light at the nanoscale. A variety of applications for polymer optical nanofibers, including waveguides, light sources and sensors, have been reported in recent years. In this article, the recent progress in the field of polymer optical nanofibers is reviewed in terms of their fabrication, characterization and applications. In particular, we focus on functionalized polymer nanofibers doped with functional materials, such as dye molecules, noble metal nanoparticles, quantum dots and rare earth ions, which greatly expand their capabilities of generating, propagating, converting and modulating light at the nanoscale.

Light: Science \& Applications (2013) 2, e102; doi:10.1038/lsa.2013.58; published online 11 October 2013

Keywords: functionalization; polymer nanofibers; waveguides

\section{INTRODUCTION}

Polymer optical fibers, also referred to as plastic optical fibers, have generated increasing research interest in recent years. ${ }^{1-5}$ Because of their unique merits, including easy handling, low cost, infrared (longwavelength) transparency, great flexibility and excellent biocompatibility, polymer optical fibers have great potential for short-distance (in-building) networking, optical sensing and power delivery. Current trends in miniaturizing electronic circuits and components to their ultimately fundamental forms have spurred great efforts in reducing the photonic structures and components to scales comparable to or less than the wavelength of light. As potential building blocks for photonic circuitry and integration, polymer waveguides in the form of free-standing optical fibers, when being shrunk to polymer nanofibers with diameters of tens or hundreds of nanometers, ${ }^{6-9}$ have been attracting increasing attention as versatile platforms for manipulating light at the nanoscale. ${ }^{8-34}$ Compared to their micrometer-scale counterparts, polymer nanofibers that function as subwavelength optical waveguides show many amazing characteristics, ${ }^{35,36}$ such as tight optical confinement, a high fraction of evanescent fields, an ultrasmall allowable bending radius, fast molecular diffusion or evaporation and a small footprint. Meanwhile, compared with nanofibers or nanowires composed of inorganic materials (e.g., glasses or semiconductors), ${ }^{37-39}$ polymer nanofibers exhibit fascinating properties: first, the polymer matrix is hospitable to a variety of functional dopants, ranging from dye molecules and quantum dots to noble metal nanoparticles, which can be readily employed to tailor the optical, electrical and magnetic properties of the host nanofibers with great versatility. Secondly, polymer nanofibers have a permselective nature with respect to gas molecules, high surface-to-volume ratios and high flexibility of surface functionalities, offering more possibilities for polymer nanofibers in applications such as optical sensing. In addition, polymer nanofibers exhibit excellent mechanical flexibility and biocompatibility, as well as simple, low-cost fabrication. To date, polymer optical nanofibers have been successfully applied in nanometer-scale waveguides, ${ }^{7-9,11-13}$ incoherent light-emitting devices, ${ }^{14-23,33,34}$ lasers, ${ }^{10,24-29}$ optical sensors ${ }^{8,22,30-32}$ and photodetectors, ${ }^{40}$ and they are expected to be an increasingly active platform for manipulating light in future nanophotonic fields.

In this article, we review the recent progress on the fabrication, characterization and applications of polymer optical nanofibers, with special emphasis on polymer nanofibers functionalized with dopants, including dye molecules, quantum dots, noble metal nanoparticles and rare earth ions.

\section{FABRICATION OF POLYMER NANOFIBERS}

A number of techniques, including chemical synthesis, ${ }^{41-43}$ nanolithography, ${ }^{44,45}$ electrospinning ${ }^{6,10,11,46}$ and physical drawing, ${ }^{7-9,22,31,32,34}$ have currently been developed for the fabrication of polymer nanofibers. Among these techniques, physical drawing is an optimal method for fabricating polymer nanofibers with excellent surface qualities that are highly desired for low-loss optical waveguiding.

In a typical physical drawing fabrication, a sharp tip (e.g., a tungsten probe $^{8,31}$ or tapered fiber probe ${ }^{9,12}$ ) is used to directly draw polymer nanofibers out of a droplet of polymer solution or melt cast onto a glass slide. Using this technique, optical-quality polymer nanofibers of polystyrene (PS), poly(methyl methacrylate) (PMMA), polyacrylamide (PAM), poly(vinyl alcohol) (PVA) and poly(ethylene oxide) (PEO) have been fabricated with high uniformity and excellent surface smoothness. ${ }^{8,12,16,22,31}$ Usually, the diameter of the as-drawn polymer 
nanofibers can be roughly controlled by the drawing speed and the solution concentration.

Electrospinning is another versatile technique for fabricating polymer nanofibers from a broad range of polymer materials that has the capacity for high-volume production. ${ }^{6,10,11,46}$ This technique uses high-voltage electric fields applied between a metallic needle and a conductive collector to spin polymer solutions or melts into fibers with micro- or nanometer diameters and is capable of fabricating a single nanofiber with a length up to kilometers (corresponding to an aspect ratio of larger than $10^{9}$ ). Additionally, the electrospun polymer nanofibers can be directly collected as uniaxially aligned arrays by properly designing the conductive collector, ${ }^{47}$ making it possible to pattern the nanofibers during the fabrication process.

To functionalize the polymer nanofibers, the simplest and most commonly used method is to add functional dopants into the polymer solution in the initial step and then solidify the dopants into the nanofiber during the drawing process. A variety of functional dopants, including dye molecules, ${ }^{10,14,16,27}$ quantum dots, ${ }^{11,19,20,22}$ noble metal nanoparticles, ${ }^{31}$ rare earth ions, ${ }^{23}$ carbon-based materials, ${ }^{48-50}$ biomaterials ${ }^{51,52}$ and chemical indicators, ${ }^{8}$ have been successfully doped into polymer nanofibers. Figure 1 presents microscope images of typical functionalized polymer nanofibers doped with dye molecules (Figure 1a), CdSe quantum dots (Figure 1b), gold nanorods (GNRs) (Figure 1c), a europium complex (Figure 1d), a silver nanowire (Figure 1e) and graphene (Figure 1f). Due to the hospitability of the polymer matrix, the exotic dopants do not usually lead to serious degradation of the polymer nanofibers in terms of the structural integrity and geometric uniformity. ${ }^{8,16,22,31}$

\section{MICROMANIPULATION}

To integrate as-drawn polymer nanofibers into nanophotonic circuits or devices, micromanipulation techniques are desired for tailoring and assembling these tiny building blocks into functional structures or geometries. ${ }^{12,53-56}$ Using precisely controlled tungsten or tapered fiber probes with tip sizes of tens to hundreds of nanometers, polymer nanofibers can be cut, picked up, transferred, bent and shaped under an optical microscope.

As shown in Figure 2a, to intercept a section of polymer nanofiber with a desired length from a polymer nanofiber, an electrochemically sharpened tungsten probe mounted on a three-dimensional translation stage is used to cut the polymer nanofiber at the desired point. The intercepted polymer nanofiber is then picked up (Figure 2b), transferred and deposited (Figure 2c) onto a certain substrate (e.g., a low-index $\mathrm{MgF}_{2}$ wafer or silica aerogel) using a tapered fiber probe (drawn from a standard glass optical fiber ${ }^{37}$ ) for nanophotonic integration. When deposited on a certain substrate with a smooth surface, polymer nanofibers can be firmly held in position by van der Waals forces and electrostatic interactions between the nanofiber and the substrate. Using directional pushing or dragging operations on the substrate surface against the friction force with micromanipulation probes, polymer nanofibers can be bent and assembled into desired structures or patterns (Figure $2 \mathrm{~d}$ and $2 \mathrm{e}$ ). A variety of nanofiber-based
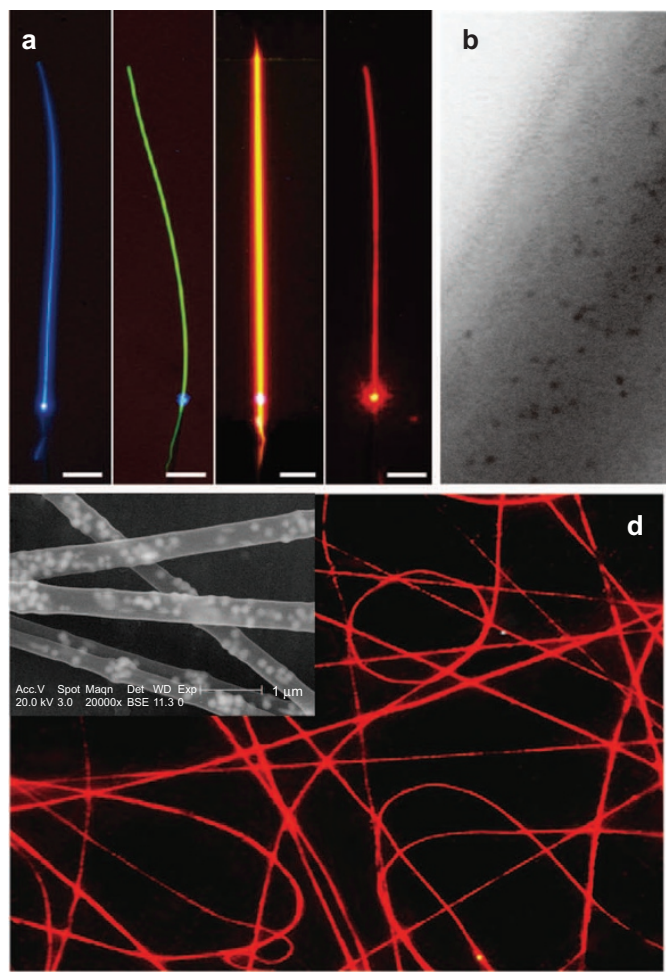

d

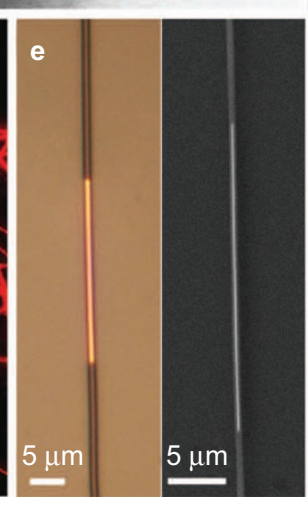

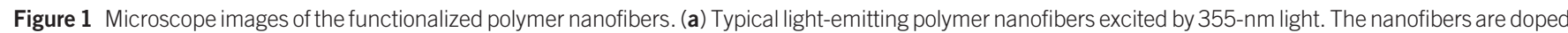

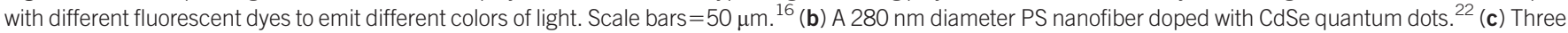

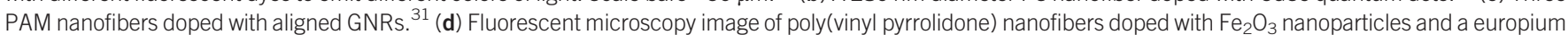



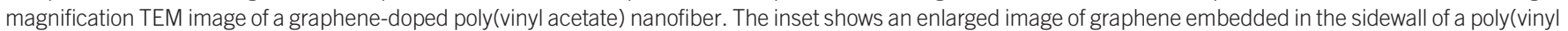

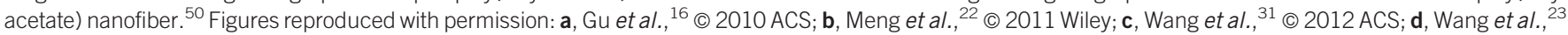


electron microscope. 


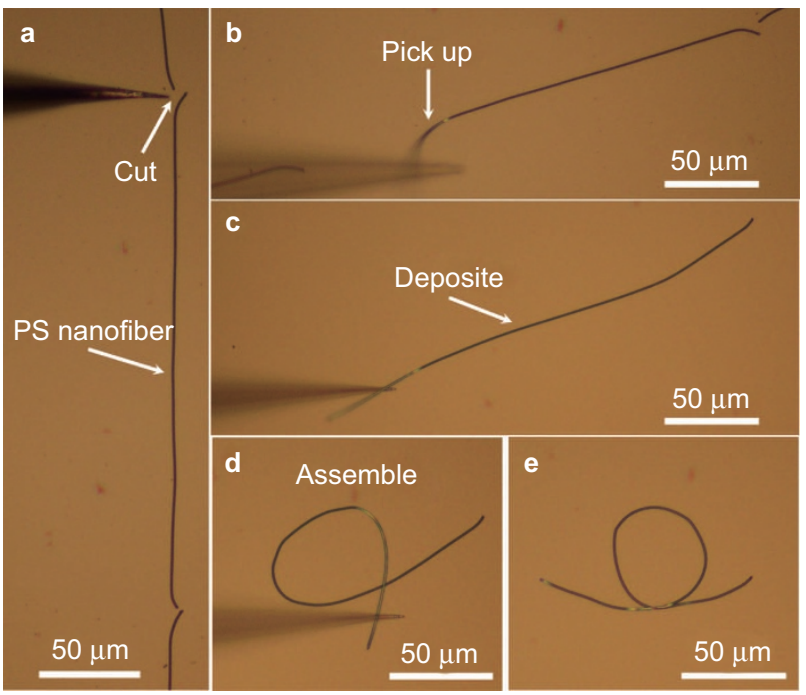

Figure 2 Micromanipulation of single nanofibers. (a) Cutting a PS nanofiber using a tungsten probe. (b) Picking up the cut PS nanofiber using a tapered fiber. (c) Depositing the PS nanofiber on a $\mathrm{MgF}_{2}$ wafer. (d, e) Assembling the PS nanofiber into a ring structure. PS, polystyrene.

photonic components or devices that use micromanipulation, e.g., microcouplers, ${ }^{12,53}$ resonators ${ }^{12,55}$ and interferometers, ${ }^{56}$ have been reported.

\section{OPTICAL PROPERTIES}

For nanophotonic applications, the optical properties, especially the waveguiding capabilities, are among the most important properties of polymer nanofibers. Here, we briefly review the principles of optical waveguiding, evanescent coupling and optical losses of polymer nanofibers.

\section{Basic model}

The mathematical model of a polymer nanofiber is shown in Figure $3 \mathrm{a}$. The refractive indices of the polymer and the surrounding medium are $n_{1}$ and $n_{2}$, respectively, and the diameter of the polymer nanofiber is $2 a$. The index profile of the waveguiding nanofiber is expressed as

$$
n(r)= \begin{cases}n_{1}, & 0<r<a \\ n_{2}, & a \leq r<\infty\end{cases}
$$

For non-dissipative polymers, the waveguiding properties of a polymer nanofiber can be retrieved by analytically solving the following Helmholtz equations:

$$
\begin{aligned}
& \left(\nabla^{2}+n^{2} k^{2}-\beta^{2}\right) \vec{e}=0, \\
& \left(\nabla^{2}+n^{2} k^{2}-\beta^{2}\right) \vec{h}=0
\end{aligned}
$$

where $k=2 \pi / \lambda, \lambda$ is the wavelength of the light in a vacuum and $\beta$ is the propagation constant.

Benefiting from the circular cross-section of the polymer nanofiber, Equation (2) can be analytically solved in cylindrical coordinates ${ }^{35}$ with the eigenvalue equations shown as follows:

$H E_{v m}$ and $E H_{v m}$ modes

$$
\begin{aligned}
& \left\{\frac{J_{v}^{\prime}(U)}{U J_{v}(U)}+\frac{K_{v}^{\prime}(W)}{W K_{v}(W)}\right\}\left\{\frac{J_{v}^{\prime}(U)}{U J_{v}(U)}+\frac{n_{2}^{2} K_{v}^{\prime}(W)}{n_{1}^{2} W K_{v}(W)}\right\}= \\
& \left(\frac{v \beta}{k n_{1}}\right)^{2}\left(\frac{V}{U W}\right)^{4}
\end{aligned}
$$

$T E_{O m}$ modes

$$
\frac{J_{1}(U)}{U J_{0}(U)}+\frac{K_{1}(W)}{W K_{0}(W)}=0
$$

and $T M_{O m}$ modes

$$
\frac{n_{1}^{2} J_{1}(U)}{U J_{0}(U)}+\frac{n_{2}^{2} K_{1}(W)}{W K_{0}(W)}=0
$$

where $J_{v}$ is the Bessel function of the first kind, $K_{v}$ is the modified Bessel function of the second kind and $U=a\left(k_{0}^{2} n_{1}^{2}-\beta^{2}\right)^{1 / 2}$, $W=a\left(\beta^{2}-k_{0}^{2} n_{2}^{2}\right)^{1 / 2}, V=k_{0} a\left(n_{1}^{2}-n_{2}^{2}\right)^{1 / 2}$.

By numerically solving Equations (3)-(5), the waveguiding modes $(\beta)$ supported by the polymer nanofiber can be obtained. For reference, Figure $3 \mathrm{~b}$ shows $\beta$ of the three lowest-order waveguiding modes $\left(H E_{11}, T E_{01}\right.$ and $\left.T M_{01}\right)$ of a PS nanofiber (refractive index: $\sim 1.59$ ) waveguiding $660 \mathrm{~nm}$ light. It shows that, to operate a PS nanofiber in single mode (for $660 \mathrm{~nm}$ light in air or vacuum), which
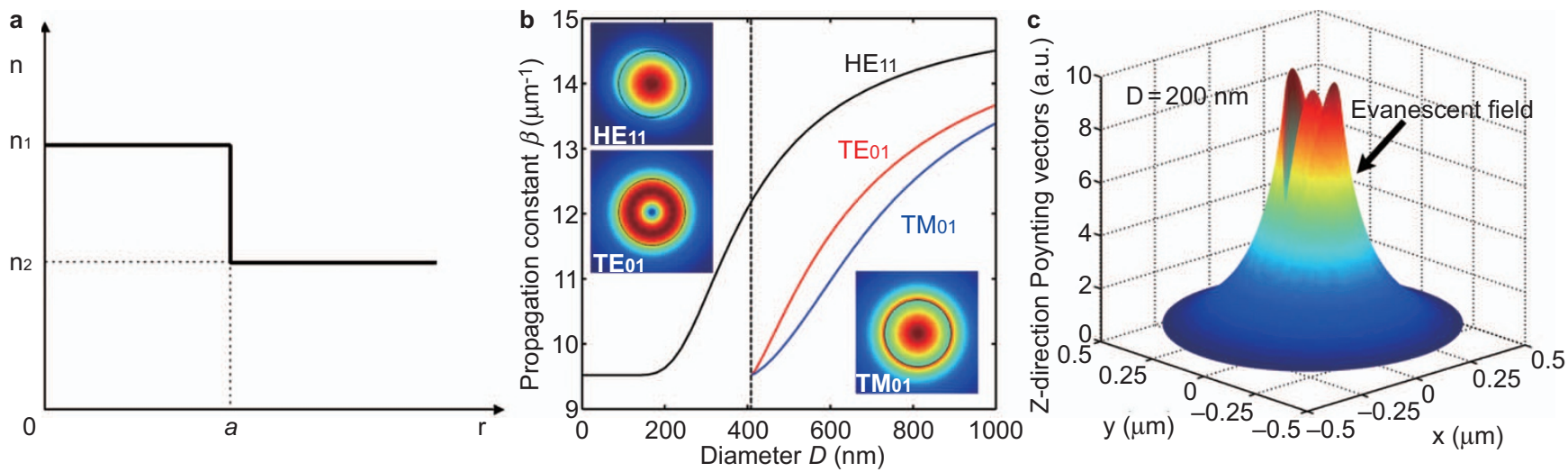

Figure 3 Mathematical modeling of an optical nanofiber. (a) Index profile of a nanofiber. (b) Numerical solutions of the propagation constant ( $\beta$ ) of an air-clad PS nanofiber at $660 \mathrm{~nm}$. Dotted line, critical diameter for single-mode operation. Insets, power distributions (Poynting vectors) of the three modes at the transverse crossplane of a $600 \mathrm{~nm}$ diameter PS nanofiber. (c) Calculated Poynting vectors of a $200 \mathrm{~nm}$ diameter PS nanofiber guiding 660-nm light. PS, polystyrene. 
is required in most applications, the diameter of the fiber should be less than $410 \mathrm{~nm}$. Compared to their counterparts with large diameters, polymer nanofibers with subwavelength diameters offer many fascinating properties, such as tight optical confinement (insets of Figure 3b), a large fraction of evanescent fields (Figure 3c) and abnormal waveguiding dispersions, which open up new opportunities for guiding and manipulating light at the nanoscale.

\section{Evanescent coupling}

One considerable challenge in realizing polymer nanofiber-based nanophotonic components and devices is efficiently coupling light into polymer nanofibers with subwavelength-scale endfaces. While the conventional lens-focused butt coupling approach is no longer valid, an evanescent coupling technique has been proven to be very efficient with a high compactness. ${ }^{8,16,57}$ As schematically illustrated in Figure $4 \mathrm{a}$, a fiber taper, which is drawn from a standard glass optical fiber, ${ }^{37}$ is placed in close contact with one end of a polymer nanofiber supported by a low-index $\mathrm{MgF}_{2}$ substrate (refractive index: $\sim 1.39$ ). Due to the strong evanescent coupling between the nanofiber and the fiber taper, ${ }^{57}$ light can be launched into the nanofiber with high efficiency (up to 90\%) (Figure 4b). Note that there exists a short-pass filtering effect for substrate-supported nanofibers due to substrateinduced leakage ${ }^{58}$ For reference, Figure $4 \mathrm{c}$ presents an optical microscope image of a $\mathrm{MgF}_{2}$-supported $360 \mathrm{~nm}$ diameter PS nanofiber waveguiding a broadband supercontinuum laser. The obvious color change in the input (white) versus output (blue) indicates wavelengthdependent transmission ${ }^{58}$ of a substrate-supported polymer nanofiber. To avoid this effect for broadband waveguiding applications, the nanofiber can be suspended across a microchannel. ${ }^{8,22,31}$ Figure $4 \mathrm{~d}$ presents the broadband transmittability of a suspended $330 \mathrm{~nm}$ diameter PMMA nanofiber, with optical microscope images of the nanofiber waveguiding broadband supercontinuum and monochromatic lasers shown in the inset. ${ }^{8}$

\section{Optical losses}

For photonic applications, optical loss is of the most relevant parameters. For polymer nanofibers, the waveguiding loss is mainly determined by material and geometric factors, including spectral absorption, surface non-uniformities, surface contamination and the surrounding media. It has been reported in previous studies ${ }^{8,22,31}$ on air-clad and straight polymer nanofibers (e.g., PMMA, PS and PAM nanofibers) that the waveguiding loss is usually less than $0.1 \mathrm{~dB} \mathrm{~mm}^{-1}$, which is acceptable in most nanophotonic applications that require an effective length of less than $1 \mathrm{~mm}$.

To construct highly compact photonic devices or circuits using polymer nanofibers, sharp bent structures ${ }^{12,37,53}$ are often required. Benefiting from the tight optical confinement of the waveguiding modes in polymer nanofibers with low-index surroundings, the additional bending loss remains low with a bending radius as low as several micrometers. For example, with a bending radius as small as $3 \mu \mathrm{m}$, the bending loss of a $350 \mathrm{~nm}$ diameter PS nanofiber at $633 \mathrm{~nm}$ is approximately $0.5 \mathrm{~dB} / 90^{\circ},{ }^{59}$ which is acceptable for photonic circuitry in most cases. In addition, because the polymer is a ductile material that presents high flexibility for bending, polymer nanofibers are good candidates for bent waveguides. ${ }^{12}$

\section{FUNCTIONALIZATION}

As a result of their favorable optical waveguiding properties, polymer nanofibers offer an attractive platform for manipulating light at the nanoscale and serve as effective building blocks for nanophotonic circuits and devices. Compared to other types of one-dimensional nanowaveguides, such as glass nanofibers or semiconductor nanowires, ${ }^{37-39}$ nanofibers made of polymer matrices possess the unique opportunity, as excellent hosts for functional materials, to greatly expand their capabilities of generating, propagating, converting and modulating light at the nanoscale. In this section, we review functionalized polymer optical nanofibers, which are categorized according to the functional dopants, including dye molecules, quantum dots, noble metal nanoparticles, rare earth ions, carbon-based materials and biomaterials.

\section{Dye molecules}

Dye molecules are commonly used organic optical gain materials for photonic applications, such as optical amplification and light emission. In the past decade, considerable attention has been focused on the fabrication of dye-doped active polymer nanofibers for potential applications in incoherent light-emitting sources, ${ }^{14-16,60,61}$ lasers $^{10,27,28}$ and optical sensors. ${ }^{62,63}$

With appropriate dye dopants (e.g., rhodamine B, rhodamine 6G, perylene and nile blue A), light-emitting polymer nanofibers with
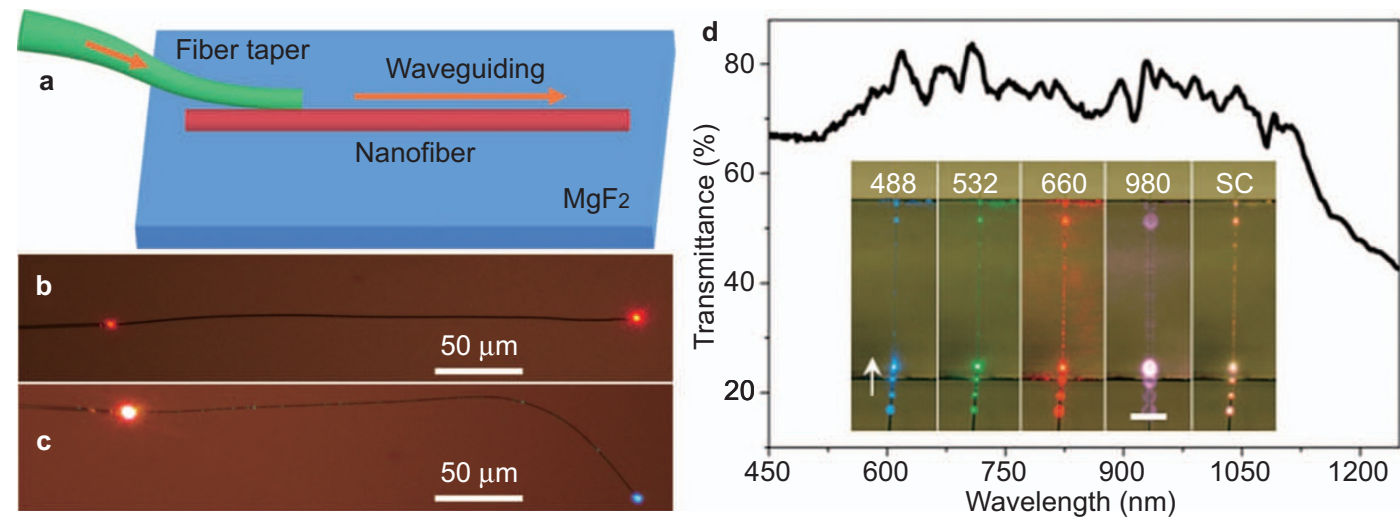



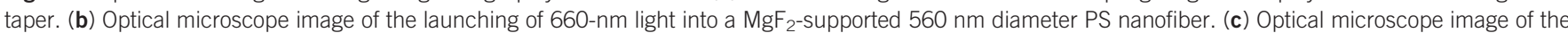
launching of a supercontinuum into a MgF 2 -Supported 360 nm diameter PS nanofiber. (d) Broadband transmission spectrum of a 330 nm diameter PMMA nanofiber.

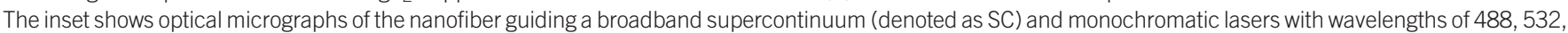
660 and $980 \mathrm{~nm}$. Scale bar $=50 \mu \mathrm{m} .{ }^{8}$ Figure reproduced with permission: d, Gu et al., ${ }^{8}$ @ 2008 ACS. PMMA, poly(methyl methacrylate); PS, polystyrene. 
tunable emission in the visible and near infrared $(<1 \mu \mathrm{m})$ spectral range can be readily obtained. ${ }^{10,14,16}$ Polymer nanofibers doped with typical dye molecules of perylene, fluorescein sodium salt, $\mathrm{Ru}$ (bpy) ${ }_{3} \mathrm{Cl}_{2}$ and zinc phthalocyanine (from left to right) are shown in Figure 1a; the emission colors of blue, green, orange and red correspond well to the characteristic emission of the four dopants. Meanwhile, by simultaneously doping multiple dye molecules with different emission bands in the initial polymer solvents, light-emitting polymer nanofibers with broadband emission ${ }^{16,64}$ can be readily fabricated. As shown in Figure $5 a$ and 5b, by codoping three different dyes (rhodamine B, perylene and zinc phthalocyanine) in the proper weight ratios, white light-emitting PS nanofibers (Figure 5b) can be obtained. ${ }^{16}$ Additionally, by controlling the molar ratio of the different dyes, it is possible to tune the emission color of the nanofiber; for example, Zhang et al. ${ }^{64}$ prepared PEO nanofibers doubly doped with a blue light emitter (1,3,5-triphenyl-2-pyrzoline, TPP) and a red light emitter (4-(dicyanomethylene)-2-methyl-6-( $p$-dimethyl-aminostyryl)$4 \mathrm{H}$-pyran, DCM) and tuned their emission color across a broad spectral range by changing the molar ratio of the dyes (Figure $5 c-5 f$ ). These color-tunable light-emitting polymer nanofibers can be exploited as nanoscale light sources and integrated into optical sensing for lab-on-chip applications. ${ }^{65}$

Usually, the dye-doped polymer nanofibers are excited using direct irradiation. ${ }^{10,14,15,27}$ To enhance the excitation efficiency and selectivity, a waveguiding excitation approach relying on high-efficiency evanescent coupling was recently developed. ${ }^{16}$ As shown in Figure 6, light from a fiber taper can be efficiently coupled into the polymer nanofiber for optical excitation (see also Figures 1a and 5a). Using this technique, the light-nanofiber interaction can be greatly enhanced compared to direct irradiation (more than 3 orders of magnitude), dramatically increasing the excitation efficiency and reducing the excitation power consumption. Additionally, using waveguiding excitation with a fiber taper, the excitation light is tightly localized around the polymer nanofibers, which allows high-density integration of these nanofibers with low crosstalk.

Under optical excitation, the ends of the dye-doped polymer nanofibers show bright spots of light, ${ }^{10,16}$ indicating that the fluorescent emission is confined and waveguided along the nanofibers. As active nanofiber waveguides, lasing action may occur if a certain optical feedback, such as end-face reflection or closed-loop recirculation, is
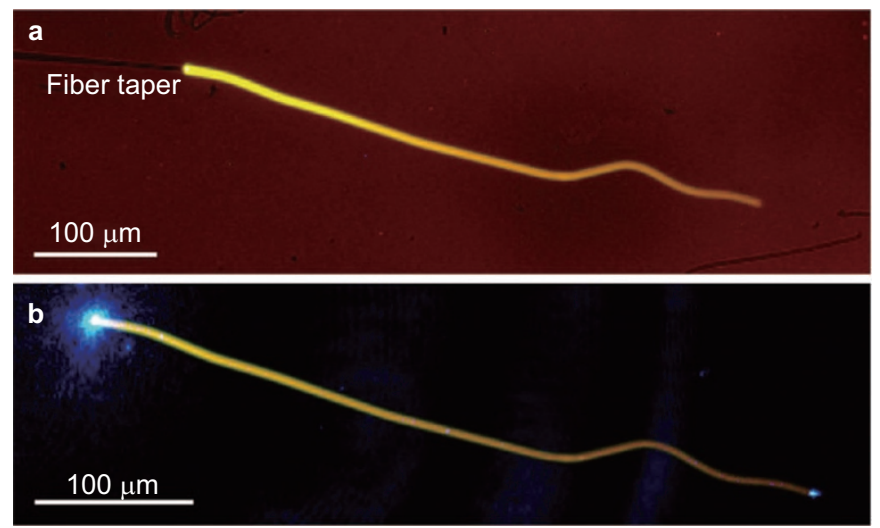

Figure 6 Photoluminescence microscope images of a 380-nm-diameter, 520$\mu \mathrm{m}$-length, rhodamine B-doped PS nanofiber excited by 473-nm light from the left side taken (a) with and (b) without the long-pass emission filter. ${ }^{16}$ Figure reproduced with permission from Gu et al., ${ }^{16}$ @ 2010 ACS. PS, polystyrene.

introduced. ${ }^{10,27,28}$ For example, Camposeo et al. ${ }^{10}$ demonstrated optically pumped lasing emission in individual electrospun PMMA nanofibers doped with laser dyes. As shown in Figure 7a, each segmented nanofiber constitutes a Fabry-Pérot cavity, with feedback provided by end-face reflection. The laser emission from the nanofiber

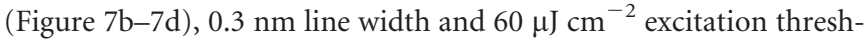
old, is comparable to the results from polymer lasers fabricated using other methods. Lasing emission in dye-doped polymer nanofiber ring structures has also been demonstrated. By assembling a dye-doped polycarbonate nanofiber into a knotted structure approximately $360 \mu \mathrm{m}$ in diameter and irradiating the structure with laser pulses, Song et al. ${ }^{27}$ reported lasing action with a $0.07 \mathrm{~nm}$ line width. The threshold fluence is relatively high $\left(\sim 2.6 \mathrm{~mJ} \mathrm{~cm}{ }^{-2}\right)$, which may be greatly reduced by using a waveguiding excitation approach.

Optical sensors based on fluorescence quenching or an emission color change of dye molecules have also been developed using dyedoped polymer nanofibers. ${ }^{62,63}$ For example, Wang et al. ${ }^{63}$ demonstrated ultrasensitive, naked eye detection of explosives based on the fluorescence quenching of a nanofibrous film. The sensing film was fabricated by electrospinning pyrene molecules with PS in the presence of an organic salt (tetrabutylammonium hexafluorophosphate). A
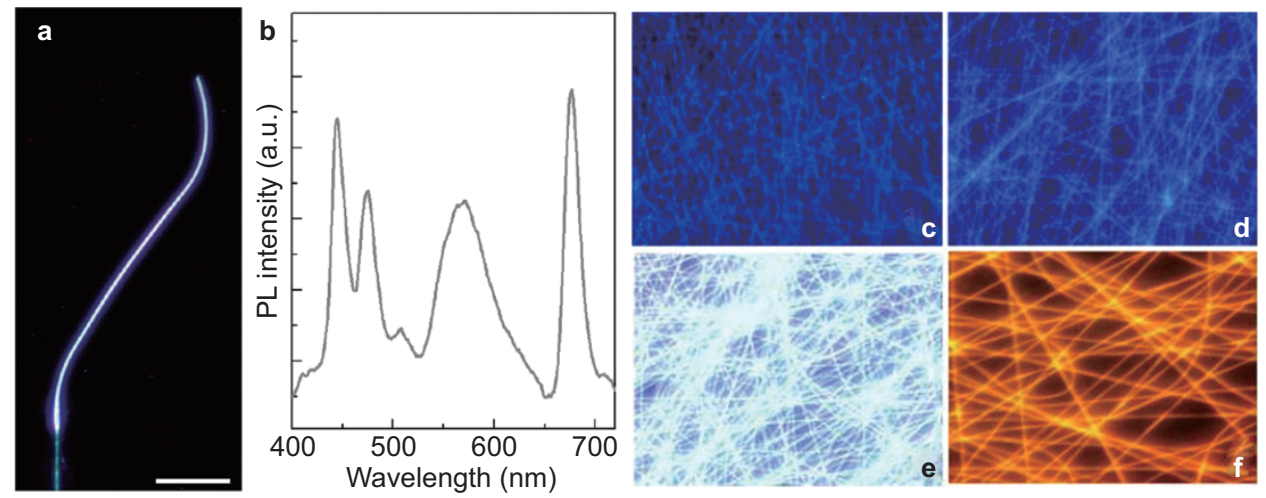

Figure 5 Optical characterization of several typical dye-doped polymer nanofibers. (a) Photoluminescence microscope image of a $430 \mathrm{~nm}$ diameter PS nanofiber codoped with rhodamine B, perylene and zinc phthalocyanine. Scale bar $=50 \mu \mathrm{m}$. (b) Photoluminescence spectrum of the doped PS nanofiber. ${ }^{16}$ Fluorescence microscopy images of (c) PEO-TPP, (d) PEO-TPP/DCM (1/0.005), (e) PEO-TPP/DCM (1/0.02) and (f) PEO-DCM nanofibers. ${ }^{64}$ Figures reproduced with permission: a, b, Gu et al., ${ }^{16}$ @ 2010 ACS; c-e, Zhang et al., ${ }^{64}$ @ 2012 Springer. DCM, 4-(dicyanomethylene)-2-methyl-6-(p-dimethyl-aminostyryl)-4H-pyran; PEO, poly(ethylene oxide); PS, polystyrene; TPP, 1,3,5-triphenyl-2-pyrzoline. 


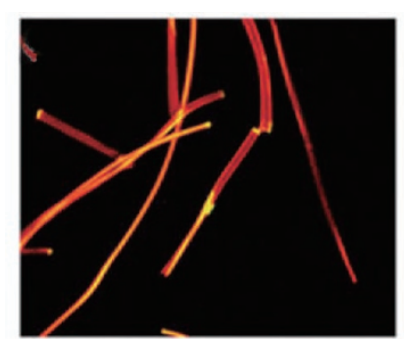

C

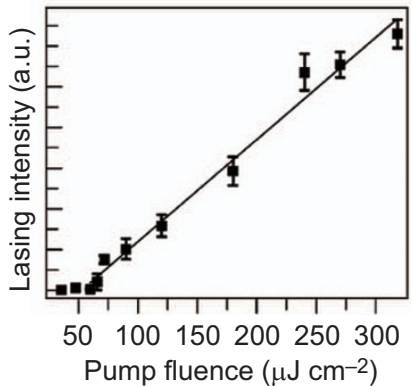

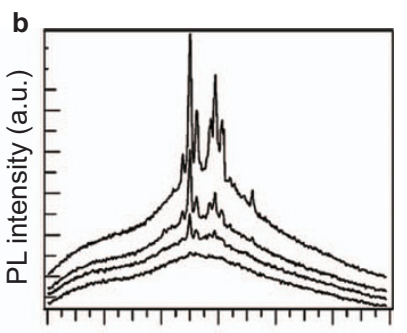

560570580590600610620 Wavelength $(\mathrm{nm})$

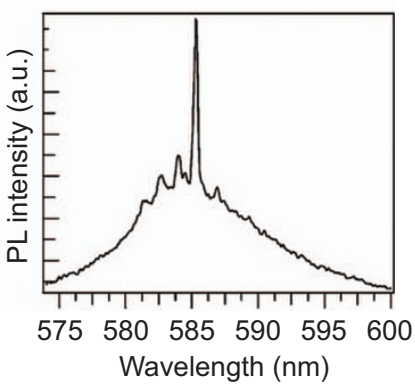

Figure 7 Optical characterization of rhodamine 6G-doped PMMA nanofibers. (a) Fluorescence image of typical nanofibers. (b) Typical emission spectra of the nanofiber under different incident pump fluences. From bottom to top: 50, 65, 70 and $100 \mu \mathrm{J} \mathrm{cm}^{-2}$. (c) Laser emission intensity of the nanofiber versus the excitation fluence. (d) Emission spectrum of a single nanofiber at a pump fluence of $150 \mu \mathrm{J} \mathrm{cm}{ }^{-2} .{ }^{10}$ Figure reproduced with permission from Camposeo et al.,${ }^{10}$ (c) 2009 Wiley. PMMA, poly(methyl methacrylate).

wide range of nitro explosives could be 'visually' detected by the vapors released from $1 \mathrm{ng}$ of explosives residues.

\section{Noble metal nanoparticles}

Noble metal nanoparticles have rich optical properties stemming from their localized surface plasmon resonance (LSPR) that can be excited by electromagnetic waves. These nanoparticles have attracted intensive research interest for a variety of emerging applications. ${ }^{66,67}$

Noble metal nanoparticles can be dispersed in polymer nanofibers without obvious aggregation,. ${ }^{31,68-75}$ with the density of nanoparticles down to the single nanoparticle level. ${ }^{31}$ Note that, as shown in Figure 1c, for nanoparticles with anisotropic geometries such as GNRs, long-range alignment with their longitudinal axis parallel to the nanofiber axis can be achieved, ${ }^{31,73-75}$ which bestows the nanofiber with collective polarization-dependent optical properties (Figure 8a$8 \mathrm{c}$ ). The unidirectional alignment is realized by shear forces exerted on the GNRs during the nanofiber drawing process. ${ }^{31,73}$ The metal dopants not only add plasmonic features but also modify the electronic properties of the polymer nanofiber, creating a new photonplasmon hybrid nanomaterial. For example, Chen et al. ${ }^{72}$ demonstrated electrospun Ag nanoparticle-doped poly( $p$-phenylenevinylene) nanofibers with a highly sensitive optoelectronic response. Under light illumination of $5.76 \mathrm{~mW} \mathrm{~cm} \mathrm{~m}^{-2}$ and a voltage of $20 \mathrm{~V}$, the photocurrent is considerably larger than the dark current for a single Ag nanoparticle-doped poly( $p$-phenylenevinylene) nanofiber. By electrospinning Ag nanosphere- or GNR-doped PVA into a nanofiber mat on a large scale with high density (Figure $8 \mathrm{~d}$ ), Yu and co-workers ${ }^{71,74}$ reported a flexible substrate for surface-enhanced Raman spectroscopy using the electrospun mat with high sensitivity, reproducibility and stability (Figure 8e).
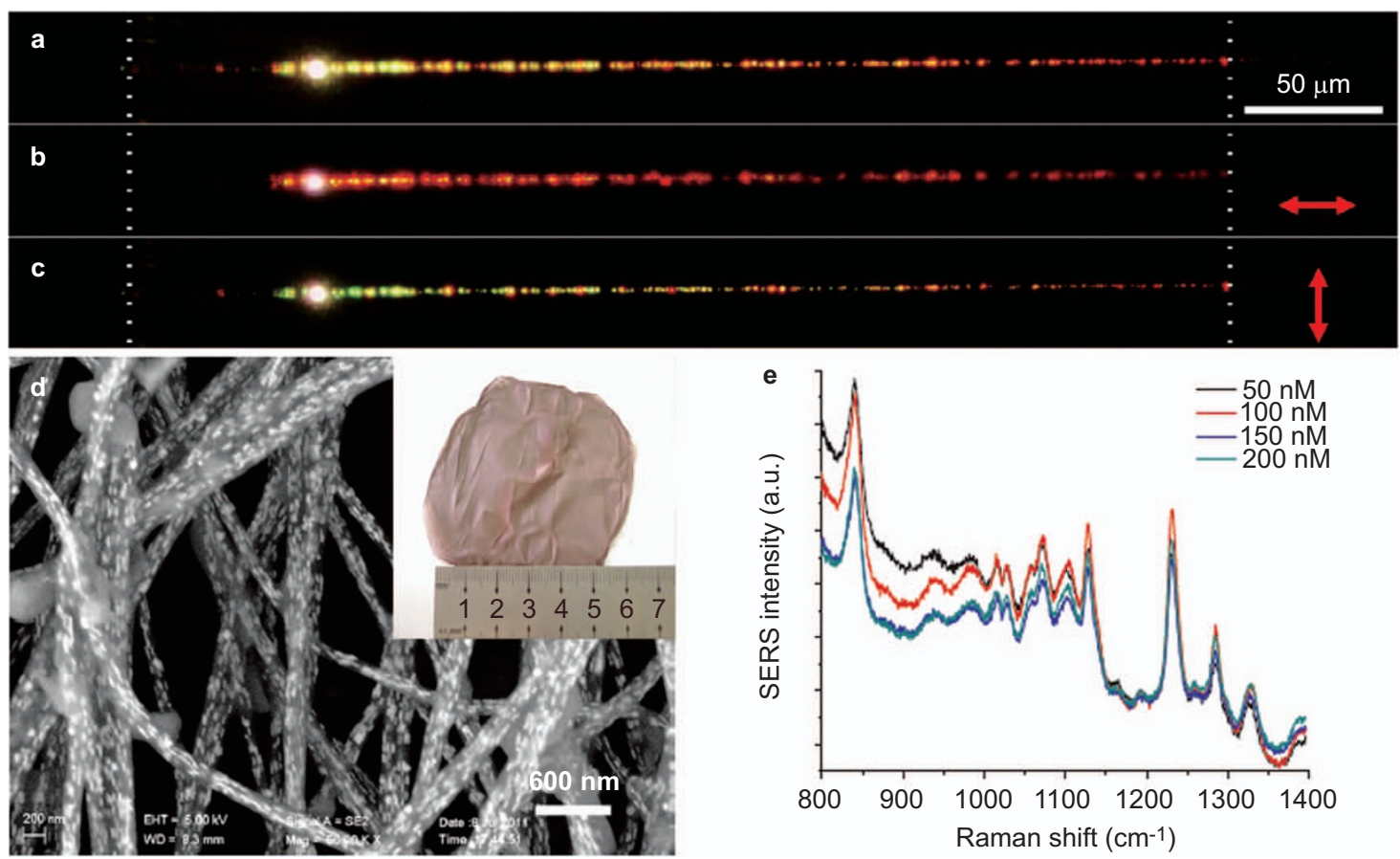

Figure 8 Optical characterization of GNR-doped polymer nanofibers. (a-c) Optical microscope images of waveguiding excitation of GNRs embedded in a PAM nanofiber $590 \mathrm{~nm}$ in diameter. The images in $\mathbf{b}$ and $\mathbf{c}$ are taken at polarizations parallel and perpendicular to the nanofiber, respectively. ${ }^{31}$ (d) Typical backscattering SEM image of the Au/PVA nanofiber mat prepared by electrospinning. Inset, photograph of the Au/PVA nanofiber mat (with Au nanorod concentration of $200 \mathrm{nM}$ ) produced by electrospinning for $1 \mathrm{~h}$. (e) SERS spectra of $10^{-4} \mathrm{M} \mathrm{DTTCI}$ molecules absorbed on the electrospun nanofiber mats produced with different concentration of Au nanorods. ${ }^{74}$ Figures reproduced with permission: a-c, Wang et al., ${ }^{31} \odot 2012$ ACS; d, e, Zhang et al.. ${ }^{74}$ ๑ 2012 Wiley. GNR, gold nanorod; PAM, polyacrylamide; PVA, poly(vinyl alcohol); SEM, scanning electron microscope; SERS, surface-enhanced Raman scattering. 
Polymer nanofibers usually provide tightly confined waveguiding modes, whose electromagnetic fields may considerably overlap with those of the plasmonic modes of the embedded noble metal nanoparticles, making it possible to achieve highly efficient photonto-plasmon conversion using the waveguiding modes of the nanofiber for LSPR excitation. Figure 8a presents optical microscope images of the waveguiding excitation of a GNR-doped PAM nanofiber. Although the excitation optical power is as low as $50 \mathrm{nW}$, light scattered from the embedded GNRs is clearly observed along the nanofiber. Because the embedded GNRs were collectively aligned, the waveguiding nanofiber showed strong polarization-dependent light scattering (Figure $8 \mathrm{~b}$ and $8 \mathrm{c}$ ). Around the peak resonance wavelength, the excitation efficiency offered by the waveguiding approach is approximately two orders of magnitudes higher than that of conventional free-space irradiation. Figure 9a shows the waveguiding excitation of a single GNR (Figure 9b) embedded in a $350 \mathrm{~nm}$ diameter PAM nanofiber, where a single bright scattering spot could be observed. The scattering spectrum of the GNR (Figure 9c) clearly shows both LSPR peaks. Due to the strong interaction between the waveguiding light and the embedded GNRs, a photon-to-plasmon conversion efficiency up to $70 \%$ for a single GNR at its longitudinal plasmonic resonance
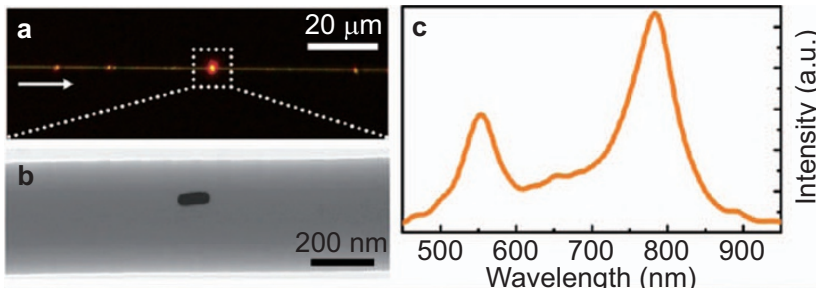

d
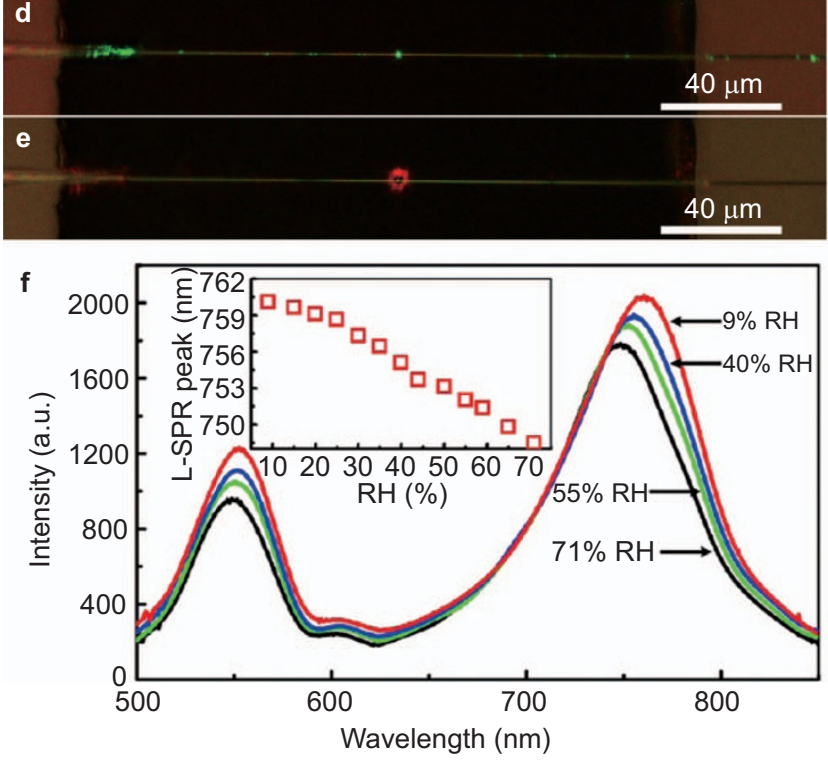

Figure 9 (a) Optical microscope image of a single GNR embedded in a waveguiding nanofiber $350 \mathrm{~nm}$ in diameter and excited by white light. (b) TEM image of the embedded GNR. (c) Scattering spectrum of the waveguiding excited GNR shown in a. (d, e) Optical microscope images of the nanofiber waveguiding monochromatic lasers with wavelengths of 532 and $785 \mathrm{~nm}$. (f) Scattering spectra of the embedded GNR exposed to air of varying $\mathrm{RH}$. Inset, the dependence of the LSPR peak on the RH of ambient air. ${ }^{31}$ Figure reproduced with permission from Wang et al.," 31 (c) 2012 ACS. GNR, gold nanorod; LSPR, localized surface plasmon resonance; $\mathrm{RH}$, relative humidity; TEM, transmission electron microscope. wavelength is obtained (Figure 9d and 9e), which is attractive for enhancing light-matter interactions within a highly localized area.

Noble metal nanoparticles are widely used in biochemical sensing because their LSPR is highly dependent on the dielectric constant of the surrounding medium. ${ }^{66}$ By measuring the spectral shift of the LSPR with respect to the refractive index change of the polymer due to the adsorption of water molecules (Figure 9f), Wang et al. ${ }^{31}$ demonstrated an optical relative humidity $(\mathrm{RH})$ sensor based on single GNRdoped PAM nanofibers with a fast response (approximately $110 \mathrm{~ms}$ ), small footprint, ultra-low optical power $(25 \mathrm{nW}$ level) and high photochemical stability.

\section{Quantum dots}

Quantum dots, usually in the form of semiconductor nanocrystals, show fascinating optical and electric properties, including the quantum confinement effect, broad absorption profiles, high optical gain and photochemical stability, and have found wide applications in areas such as photon sources, optical amplification, sensing and fluorescent labeling. ${ }^{76,77}$ To date, a variety of quantum dots have been successfully incorporated into polymer nanofibers. ${ }^{11,19-22,78-80}$ By surface modification, ${ }^{81}$ controlling the density of the quantum dots and/or rapidly evaporating the solvent during the nanofiber drawing process, ${ }^{20,22}$ the quantum dots can be well-dispersed throughout the polymer nanofibers without obvious aggregation, thereby avoiding the Förster resonance energy transfer between neighboring quantum dots and preserving the fluorescence properties of the single quantum dots.

Due to the small size of single quantum dots (typically several nanometers in diameter), the well-dispersed quantum dot dopants do not scatter waveguided light in the nanofiber, making the quantum dotfunctionalized polymer nanofibers excellent active nanowaveguides. In 2006, Liu et al. ${ }^{11}$ reported on CdSe/ZnS quantum dot-doped SU8 nanofibers fabricated by electrospinning. When the embedded quantum dots were excited by a focused laser beam, the quantum dot photoluminescence was effectively guided by the subwavelength diameter nanofibers for several micrometers. In 2011, by directly drawing doped polymer solutions, Meng et al. ${ }^{22}$ demonstrated optical-quality CdSe/ZnS quantum dot-doped PS nanofibers with a quantum dot concentration up to $10^{4} \mu \mathrm{m}^{-3}$. Launched by evanescent coupling, the nanofibers showed a low waveguiding loss $\left(<0.2 \mathrm{~dB} \mathrm{~mm}^{-1}\right.$ at $672 \mathrm{~nm}$ ) at wavelengths away from the absorption band of the quantum dots, which suggests that it is possible to efficiently guide light for millimeters (Figure 10a). Under waveguiding excitation, the nanofibers showed excellent photoluminescence properties, including highly efficient absorption and strong photoluminescence emission (Figure $10 \mathrm{~b}$ and 10c). For reference, with a quantum dot concentration of approximately $0.18 \mathrm{wt}-\%$, the measured absorption coefficient of a $430 \mathrm{~nm}$ diameter quantum dot/PS nanofiber at $532 \mathrm{~nm}$ was $\sim 31 \mathrm{~cm}^{-1}$ (Figure 10c). Additionally, compared with dye-doped polymer nanofibers, quantum dot-doped PS nanofibers show a much higher resistance to photobleaching (Figure 10d), which makes them good candidates for long-term and high-stability applications.

Because of their high surface-to-volume ratios and surface chemistry-dependent photoluminescent properties, quantum dots are highly sensitive to various chemical species and are thus promising as sensors for a variety of analytes by optical means. For example, by measuring the intensity of the waveguided photoluminescence in a single quantum dot/PS nanofiber, Meng et al. ${ }^{22}$ demonstrated a quantum dot/PS nanofiber optical sensor for RH detection with a low optical power (100 pW level) and fast response. 

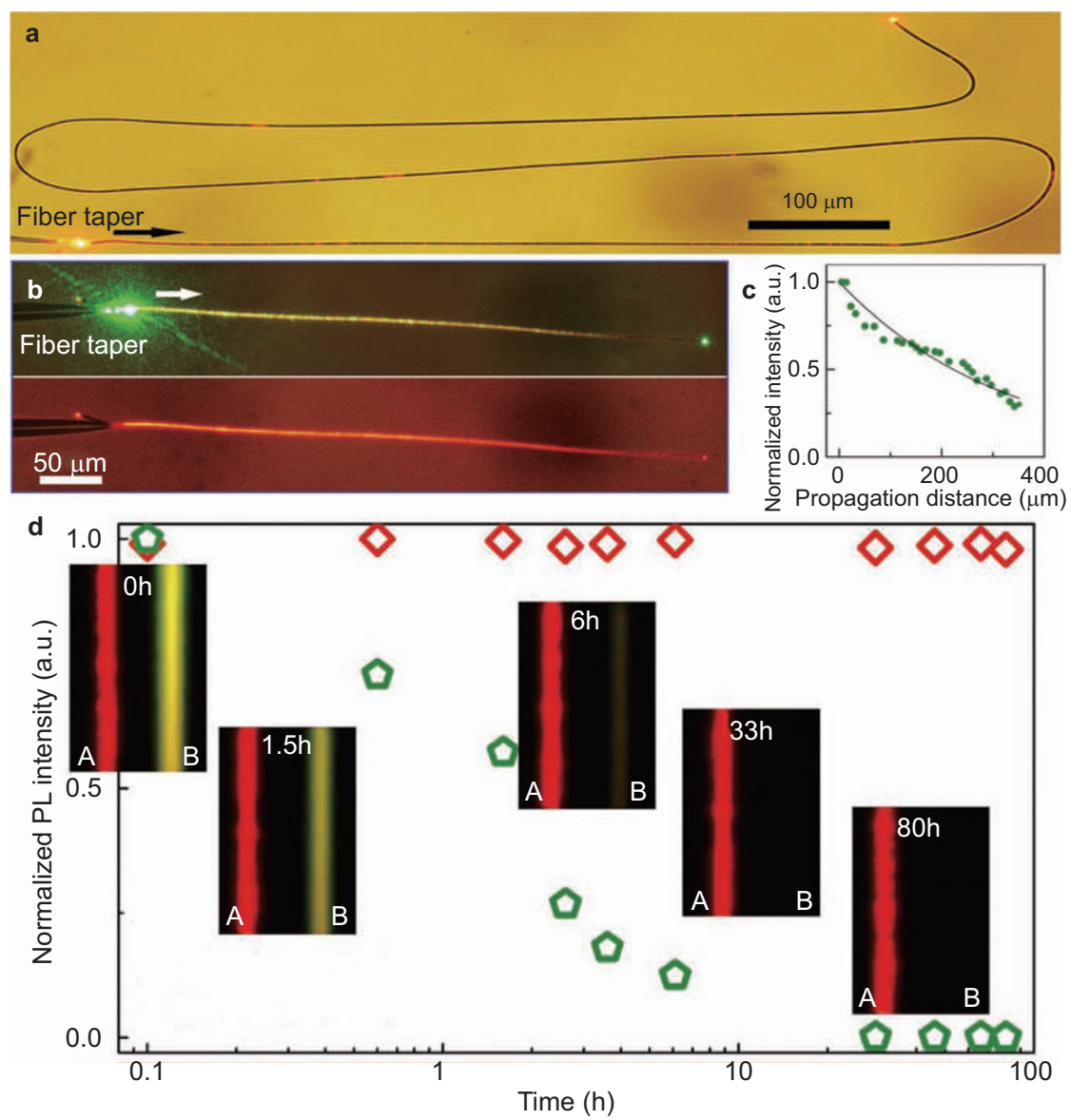

Figure 10 Optical characterization of quantum dot-doped PS nanofibers. (a) Optical microscope image of a single 560 nm diameter quantum dot/PS nanofiber guiding $672 \mathrm{~nm}$ light launched from the bottom left end. (b) Photoluminescence microscope images of a 430-nm-diameter, 380- $\mu \mathrm{m}$-long, quantum dot/PS nanofiber without (top) and with (bottom) a 532-nm notch filter. The nanofiber is excited by $532 \mathrm{~nm}$ light from the left side. (c) Propagation distance-dependent photoluminescent intensities of the nanofiber in b. (d) Time-dependent decay of the photoluminescence of a 580-nm-diameter, 160- $\mu$ m-long, quantum dot/PS nanofiber (red diamond) and a 530-nm-diameter, 160- $\mu$ m-long, rodamine 6G/PS nanofiber (green pentagon). Inset: photoluminescence images of the quantum dot/PS nanofiber (A) and rodamine 6G/PS nanofiber (B) after different time intervals. ${ }^{22}$ Figure reproduced with permission from Meng et al., 22 (c) 2011 Wiley. PS, polystyrene.

\section{Rare earth ions}

Owing to their fascinating properties, including narrow emission bands, high quantum yields, high chemical stability and long lifetimes, rare earth ions have also been considered as functional dopants for polymer nanofibers. Generally, directly doping these ions into polymer solutions is difficult due to the immiscibility of rare earth salts with organic solvents. To solve this problem, rare earth ions are often encapsulated by appropriate organic ligands to generate stable complexes that can be dissolved into polymer solutions. These complex structures also allow for heavy doping of rare earth ions without suffering from concentration quenching effects, which is attractive for achieving high gain within a short length for photonic applications in lasers and amplifiers.

To date, a number of rare earth ions, including $\mathrm{Eu}^{3+}, \mathrm{Er}^{3+}, \mathrm{Nd}^{3+}$ and $\mathrm{Yb}^{3+}$, have been successfully doped into polymer solutions and fabricated into polymer nanofibers. ${ }^{23,82-87}$ These polymer nanofibers show improved photoluminescence and thermal properties over the pure complex. In contrast to polymer nanofibers doped with organic dyes or quantum dots, which are usually optically active only at wavelengths shorter than $1 \mu \mathrm{m},{ }^{16,22}$ polymer nanofibers doped with rare earth ions offer the possibility of working in the second and third communication windows (1300 and $1500 \mathrm{~nm}),{ }^{84,86}$ which are of great interest for telecommunication applications (e.g., optical amplifiers and lasers ${ }^{88}$ ). In addition, continuous wave amplification and laser emission is possible when using rare earth ions as dopants. ${ }^{89,90}$

\section{More functional dopants}

In addition to the above-mentioned functional dopants, some other materials have also been incorporated into polymer nanofibers. For example, in 2008, Gu et al. ${ }^{8}$ doped bromothymol blue (a typical chemical indicator) into PMMA nanofibers and used the nanofiber as a fastresponse optical gas sensor for $\mathrm{NH}_{3}$ detection with a detection limit as low as $1 \mathrm{ppm}$. In 2004, Cheng et al. ${ }^{4,49}$ doped carbon nanotubes into polyacrylonitrile nanofibers to align the carbon nanotubes and improve the mechanical and electronic properties of the nanofibers. More recently, using an electrospinning technique, Bao et al. ${ }^{50}$ successfully fabricated graphene-doped poly(vinyl acetate) nanofibers (Figure 1f), with ultrafast saturable absorption and relaxation responses for photonic applications. In addition, biomaterials such as enzymes ${ }^{51}$ and $\mathrm{DNA}^{52}$ can also be used to activate polymer nanofibers for biophotonic applications, which may greatly extend the reach of polymer optical nanofibers.

\section{SUMMARY AND OUTLOOK}

So far we have reviewed the recent progress in the fabrication, characterization and application of polymer optical nanofibers, with a 
special emphasis on the doping of these polymer nanofibers with a variety of functional materials. Thanks to their outstanding merits, inherited from polymer matrices and subwavelength optical waveguiding, polymer nanofibers are very promising for a variety of photonic applications, including nanometer-scale waveguides, lightemitting devices, optical sensors and photodetectors. However, for practical applications, there are still many challenges to be addressed. For example, how can the polymer nanofibers be protected from environmental contamination? How can we precisely control the concentration and distribution of the functional dopants in the nanofibers? How to increase the long-term stability of a polymer nanofiber with relatively high power? And finally, compared with glass nanofibers, ${ }^{37}$ reducing optical losses of polymer nanofibers is always desirable. Despite these challenges, as a versatile platform merging fiber optics and nanotechnology for studying and manipulating light at nanoscale, functionalized polymer optical nanofibers will continue to find use in the generation, propagation, conversion and modulation of light for future photonic applications.

\section{ACKNOWLEDGMENTS}

This work was supported by the National Basic Research Program of China (No. 2013CB328703), the National Natural Science Foundation of China (No. 61036012) and Fundamental Research Funds for the Central Universities.

1 Zubia J, Arrue J. Plastic optical fibers: an introduction to their technological processes and applications. Opt Fiber Technol 2001; 7: 101-140.

2 Kuriki K, Koike Y. Plastic optical fiber lasers and amplifiers containing lanthanide complexes. Chem Rev 2002; 102: 2347-2356.

3 Koike Y, Asai M. The future of plastic optical fiber. NPG Asia Mater 2009; 1: 22-28.

4 Koike Y, Koike K. Progress in low-loss and high-bandwidth plastic optical fibers. J Polym Sci Pol Phys 2011; 49: 2-47.

5 Peters K. Polymer optical fiber sensors-a review. Smart Mater Struct 2011; 20: 013002.

6 Li D, Xia YN. Electrospinning of nanofibers: reinventing the wheel? Adv Mater 2004; 16: 1151-1170.

7 Harfenist SA, Cambron SD, Nelson EW, Berry SM, Isham AW et al. Direct drawing of suspended filamentary micro- and nanostructures from liquid polymers. Nano Let 2004; 4: 1931-1937.

8 Gu FX, Zhang L, Yin XF, Tong LM. Polymer single-nanowire optical sensors. Nano Lett 2008; 8: 2757-2761

9 Xing XB, Zhu H, Wang YQ, Li BJ. Ultracompact photonic coupling splitters twisted by PTT nanowires. Nano Lett 2008; 8: 2839-2843.

10 Camposeo A, Benedetto FD, Stabile R, Never AAR, Cingolani R et al. Laser emission from electrospun polymer nanofibers. Small 2009; 5: 562-566.

11 Liu HQ, Edel JB, Bellan LM, Craighead HG. Electrospun polymer nanofibers as subwavelength optical waveguides incorporating quantum dots. Small 2006; 2 . 495-499.

12 Yang Q, Jiang XS, Gu FX, Ma Z, Zhang JY et al. Polymer micro or nanofibers for optical device applications. J App/ Polym Sci 2008; 110: 1080-1084.

13 Yu HQ, Liao DW, Johnston MB, Li BJ. All-optical full-color displays using polymer nanofibers. ACS Nano 2011; 5: 2020-2025.

14 Camposeo A, Benedetto FD, Stabile R, Cingolani R, Pisignao D. Electrospun dyedoped polymer nanofibers emitting in the near infrared. Appl Phys Lett 2007; 90: 143115

15 Pagliara S, Camposeo A, Benedetto FD, Polini A, Mele E et al. Study of optical properties of electrospun light-emitting polymer fibers. Superlattices Microstruct 2010; 47: 145-149.

16 Gu FX, Yu HK, Wang P, Yang ZY, Tong LM. Light-emitting polymer single nanofibers via waveguiding excitation. ACS Nano 2010; 4: 5332-5338.

17 Benedetto FD, Camposeo A, Pagliara S, Mele E, Persano L et al. Patterning of lightemitting conjugated polymer nanofibers. Nat Nanotechnol 2008; 3: 614-619.

18 Camposeo A, Persano L, Pisignano D. Light-emitting electrospun nanofibers for nanophotonics and optoelectronics. Macromol Mater Eng 2013; 298: 487-503.

19 Sui XM, Shao CL, Liu YC. White-light emission of polyvinyl alcohol/ZnO hybrid nanofibers prepared by electrospinning. Appl Phys Lett 2005; 87: 113115.

20 Li MJ, Zhang JH, Zhang H, Liu YF, Wang CL et al. Electrospinning: a facile method to disperse fluorescent quantum dots in nanofibers without Förster resonance energy transfer. Adv Funct Mater 2007; 17: 3650-3656.

21 Benedetto FD, Camposeo A, Persano L, Laera AM, Piscopiello E et al. Light-emitting nanocomposite CdS-polymer electrospun fibres via in situ nanoparticle generation. Nanoscale 2011; 3: 4234-4239.
22 Meng C, Xiao Y, Wang P, Zhang L, Liu YX et al. Quantum-dot-doped polymer nanofibers for optical sensing. Adv Mater 2011; 23: 3770-3774.

23 Wang HG, Li YX, Sun L, Li YC, Wang W et al. Electrospun novel bifunctional magneticphotoluminescent nanofibers based on $\mathrm{Fe}_{2} \mathrm{O}_{3}$ nanoparticles and europium complex. J Colloid Interface Sci 2010; 350: 396-401.

24 Quochi F, Cordella F, Mura A, Bongiovanni G, Balzer F et al. One-dimensional random lasing in a single organic nanofiber. J Phys Chem B 2005; 109: 21690-21693.

25 Quochi F, Cordella F, Mura A, Bongiovanni G, Balzer F et al. Gain amplification and lasing properties of individual organic nanofibers. App/ Phys Lett 2006; 88: 041106.

26 O'Carroll D, Lieberwirth I, Redmond G. Microcavity effects and optically pumped lasing in single conjugated polymer nanowires. Nat Nanotechnol 2007; 2: 180-184.

27 Song QH, Liu LY, Xu L. Lasing action in dye doped polymer nanofiber knot resonator. J Lightw Technol 2009; 27: 4374-4376.

28 Das AJ, Lafargue C, Lebental M, Zyss J, Narayan KS. Three-dimensional microlasers based on polymer fibers fabricated by electrospinning. App/ Phys Lett 2011; 99: 263303.

29 Li S, Jiang WF, Xu YP, George TF. Invisible cavity of a polymeric nanofiber laser. J Phys Chem C 2011; 115: 17582-17586.

30 Zhu H, Wang YQ, Li BJ. Tunable refractive index sensor with ultracompact structure twisted by poly(trimethylene terephthalate) nanowires. ACS Nano 2009; 3: 3110 3114.

31 Wang P, Zhang L, Xia YN, Tong LM, Xu X et al. Polymer nanofibers embedded with aligned gold nanorods: a new platform for plasmonic studies and optical sensing. Nano Lett 2012; 12: 3145-3150.

32 Gu FX, Yu HK, Fang W, Tong LM. Nanoimprinted polymer micro/nanofiber Bragg gratings for high-sensitivity strain sensing. IEEE Photon Technol Lett 2013; 25 22-24.

33 Benedetto FD, Mele E, Camposeo A, Athanassiou A, Cingolani R et al. Photoswitchable organic nanofibers. Adv Mater 2008; 20: 314-318.

34 Wang $\mathrm{P}$, Li ZY, Zhang L, Tong LM. Electron-beam-activated light-emitting polymer nanofibers. Opt Lett 2013; 38: 1040-1042.

35 Tong LM, Lou JY, Mazur E. Single-mode guiding properties of subwavelengthdiameter silica and silicon wire waveguides. Opt Express 2004; 12: 1025-1035.

36 Tong LM, Zi F, Guo X, Lou JY. Optical microfibers and nanofibers: a tutorial. Opt Commun 2012; 285: 4641-4647.

37 Tong LM, Gattass RR, Ashcom JB, He SL, Lou JY et al. Subwavelength-diameter silica wires for low-loss optical wave guiding. Nature 2003; 426: 816-819.

38 Law M, Sirbuly DJ, Johnson JC, Goldberger J, Saykally RJ et al. Nanoribbon waveguides for subwavelength photonics integration. Science 2004; 305: 1269 1273.

39 Yan RX, Gargas D, Yang PD. Nanowire photonics. Nat Photon 2009; 3: 569-576.

40 O'Brien GA, Quinn AJ, Tanner DA, Redmond G. A single polymer nanowire photodetector. Adv Mater 2006; 18: 2379-2383.

41 Liang WB, Martin CR. Template-synthesized polyacetylene fibrils show enhanced supermolecular order. J Am Chem Soc 1990; 112: 9666-9668.

42 Cai ZH, Lei JT, Liang WB, Menon V, Martin CR. Molecular and supermolecular origins of enhanced electronic conductivity in template-synthesized polyheterocyclic fibrils. 1. Supermolecular effects. Chem Mater 1991; 3: 960-967.

43 Cui TY, Cui F, Zhang JH, Wang JY, Huang J et al. From monomeric nanofibers to PbS nanoparticles/polymer composite nanofibers through the combined use of $\gamma$ irradiation and gas/solid reaction. J Am Chem Soc 2006; 128: 6298-6299.

44 Noy A, Miller AE, Klare JE, Weeks BL, Woods BW et al. Fabrication of luminescent nanostructures and polymer nanowires using dip-pen nanolithography. Nano Lett 2002; 2: 109-112.

45 Marco CD, Mele E, Camposeo A, Stabile R, Cingolani R et al. Organic light-emitting nanofibers by solvent-resistant nanofluidics. Adv Mater 2008; 20: 4158-4162.

46 Dzenis Y. Spinning continuous fibers for nanotechnology. Science 2004; 304: 1917 1919

47 Li D, Wang YL, Xia YN. Electrospinning nanofibers as uniaxially aligned arrays and layer-by-layer stacked films. Adv Mater 2004; 16: 361-366.

$48 \mathrm{Ge} \mathrm{JJ}, \mathrm{Hou} \mathrm{HQ}, \mathrm{Li}$ Q, Graham MJ, Greiner A et al. Assembly of well-aligned multiwalled carbon nanotubes in confined polyacrylonitrile environments: electrospun composite nanofiber sheets. J Am Chem Soc 2004; 126: 15754-15761.

$49 \mathrm{Hou} \mathrm{HQ}, \mathrm{Ge} \mathrm{JJ}$, Zeng J, Li Q, Reneker DH et al. Electrospun polyacrylonitrile nanofibers containing a high concentration of well-aligned multiwall carbon nanotubes. Chem Mater 2005; 17: 967-973.

50 Bao QL, Zhang H, Yang JX, Wang S, Tang DY et al. Graphene-polymer nanofiber membrane for ultrafast photonics. Adv Funct Mater 2010; 20: 782-791.

51 Herricks TE, Kim SH, Kim JB, Li D, Kwak JH et al. Direct fabrication of enzymecarrying polymer nanofibers by electrospinning. J Mater Chem 2005; 15: $3241-$ 3245.

52 Bellan LM, Cross JD, Strychalski EA, Moran-Mirabal J, Craighead HG. Individually resolved DNA molecules stretched and embedded in electrospun polymer nanofibers. Nano Lett 2006; 6: 2526-2530.

53 Tong LM, Lou JY, Gattass RR, He SL, Chen XW et al. Assembly of silica nanowires on silica aerogels for microphotonic devices. Nano Lett 2005; 5: 259-262.

54 Neves AA, Camposeo A, Pagliara S, Saija R, Borghese F et al. Rotational dynamics of optically trapped nanofibers. Opt Express 2010; 18: 822-830.

55 Wang P, Gu FX, Zhang L, Tong LM. Polymer microfiber rings for high-sensitivity optical humidity sensing. App/ Opt 2011; 50: G7-G10.

56 Li YH, Tong LM. Mach-Zehnder interferometers assembled with optical microfibers or nanofibers. Opt Lett 2008; 33: 303-305. 
57 Huang KJ, Yang SY, Tong LM. Modeling of evanescent coupling between two parallel optical nanowires. Appl Opt 2007; 46: 1429-1434.

58 Chen Y, Ma Z, Yang, Q, TongLM. Compact optical short-pass filters based on microfibers. Opt Lett 2008; 33: 2565-2567.

59 Yu HK, Wang SS, Fu J, Qiu M, Li YH et al. Modeling bending losses of optical nanofibers or nanowires. Appl Opt 2009; 48: 4365-4369

60 Lee JK, Oh JH, Kim Y, Jang J. Fabrication of photoluminescent-dye embedded poly(methyl methacrylate) nanofibers and their fluorescence resonance energy transfer properties. Adv Mater 2006; 18: 2216-2219.

61 Cucchi I, Spano F, Giovanella U, Catellani M, Varesano A et al. Fluorescent electrospun nanofibers embedding dye-loaded zeolite crystals. Small 2007; 2: 305-309.

62 Davis BW, Niamnont N, Dillon R, Bardeen CJ, Sukwattanasinitt M et al. FRET detection of proteins using fluorescently doped electrospun nanofibers and pattern recognition. Langmuir 2011; 27: 6401-6408.

63 Wang Y, La A, Ding Y, Liu YX, Lei Y. Novel signal-amplifying fluorescent nanofibers for naked-eye-based ultrasensitive detection of buried explosives and explosive vapors. Adv Funct Mater 2012; 22: 3547-3555.

64 Zhang LJ, Zhang DR, Zhan SM, Yang PP, Yang GX et al. Preparation of tunable emissive poly(ethylene oxide) nanofibers by doping with TPP and DCM. Macromol Res 2012; 20: 212-215.

65 Pagliara S, Camposeo A, Polini A, Cingolani R, Pisignano D. Electrospun lightemitting nanofibers as excitation source in microfluidic devices. Lab Chip 2009; 9: 2851-2856

66 Mayer KM, Hanfaner JH. Localized surface plasmon resonance sensors. Chem Rev 2011; 111: 3828-3857.

67 Cobley CM, Chen JY, Cho EC, Wang LV, Xia YN. Gold nanostructures: a class of multifunctional materials for biomedical applications. Chem Soc Rev 2011; 40: 44-56.

68 Kim GM, Wutzler A, Radusch HJ, Michler GH, Simon P et al. One-dimensional arrangement of gold nanoparticles by electrospinning. Chem Mater 2005; 17: 4949-4957.

69 Shang TC, Yang F, Zheng W, Wang C. Fabrication of electrically bistable nanofibers. Small 2006; 2: 1007-1009.

70 Wang YZ, Li YX, Sun GE, Zhang GL, Liu H et al. Fabrication of Au/PVP nanofiber composites by electrospinning. J Appl Polym Sci 2007; 105: 3618-3622.

71 He D, Hu B, Yao QF, Wang K, Yu SH. Large-scale synthesis of flexible free-standing SERS substrates with high sensitivity: electrospun PVA nanofibers embedded with controlled alignment of silver nanoparticles. ACS Nano 2009; 3: 3993-4002.

72 Chen JF, Yang PP, Wang CJ, Zhan SM, Zhang LJ et al. Ag nanoparticles/PPV composite nanofibers with high and sensitive opto-electronic response. Nanoscale Res Lett $2011 ; 6: 121$.

73 Roskov KE, Kozek KA, Wu WC, Chhetri RK, Oldenburg AL et al. Long-range alignment of gold nanorods in electrospun polymer nano/microfibers. Langmuir 2011; 27: 13965-13969.

74 Zhang CL, Lv KP, Cong HP, Yu SH. Controlled assemblies of gold nanorods in PVA nanofiber matrix as flexible free-standing SERS substrates by electrospinning. Small 2012; 8: 648-653.
75 Zhang $\mathrm{CL}$, Lv KP, Huang HT, Cong HP, Yu SH. Co-assembly of Au nanorods with Ag nanowires within polymer nanofiber matrix for enhanced SERS property by electrospinning. Nanoscale 2012; 4: 5348-5355.

76 Klimov VI, Mikhailovsky AA, Xu S, Malko A, Hollingsworth JA et al. Optical gain and stimulated emission in nanocrystal quantum dots. Science 2000; 290: 314-317.

77 Medintz IL, Uyeda HT, Goldman ER, Mattoussi H. Quantum dot bioconjugates for imaging, labeling and sensing. Nat Mater 2005; 4: 435-446.

78 Bashouti M, Salalha W, Brumer M, Zussman E, Lifshitz E. Alignment of colloidal CdS nanowires embedded in polymer nanofibers by electrospinning. Chem Phys Chem 2006; 7: 102-106.

79 Wang SG, Li YX, Wang YZ, Yang QB, Wei Y. Introducing CTAB into CdTe/PVP nanofibers enhances the photoluminescence intensity of $\mathrm{CdTe}$ nanoparticles. Mater Lett 2007; 61: 4674-4678.

80 Tatavarty R, Hwang ET, Park JW, Kwak JH, Lee JO et al. Conductive quantum dotencapsulated electrospun nanofibers from polystyrene and polystyrene-co-maleic anhydride copolymer blend as gas sensors. React Funct Polym 2011; 71: 104-108.

81 Zhang H, Wang CL, Li MJ, Zhang JH, Lu G et al. Fluorescent nanocrystal-polymer complexes with flexible processability. Adv Mater 2005; 17: 853-857.

82 Zhang H, Song HW, Yu HQ, Li SW, Bai X et al. Modified photoluminescence properties of rare-earth complex/polymer composite fibers prepared by electrospinning. App/ Phys Lett 2007; 90: 103103.

83 Zhang H, Song HW, Dong B, Han LL, Pan GH et al. Electrospinning preparation and luminescence properties of europium complex/polymer composite fibers. J Phys Chem C 2008; 112: 9155-9162.

84 Dong B, Song HW, Yu HQ, Zhang H, Qin RF et al. Upconversion properties of $\mathrm{Ln}^{3+}$ doped $\mathrm{NaYF}_{4} /$ polymer composite fibers prepared by electrospinning. J Phys Chem $C$ 2008; 112: 1435-1440.

85 Zhang XP, Wen SP, Hu S, Chen Q, Fong $\mathrm{H}$ et al. Luminescence properties of Eu(III) complex/polyvinylpyrrolidone electrospun composite nanofibers. J Phys Chem C 2010; 114: 3898-3903.

86 Sun X, Li B, Song LT, Gong J, Zhang LM. Electrospinning preparation and photophysical properties of one-dimensional (1D) composite nanofibers doped with erbium (III) complexes. J Lumin 2010; 130: 1343-1348.

87 Huang LB, Cheng LH, Yu HQ, Zhang JS, Zhou L et al. Electrospinning preparation and optical transition properties of $\mathrm{Eu}(\mathrm{DBM})_{3}$ Phen/PS fluorescent composite fibers. Opt Commun 2012; 285: 1476-1480.

88 Grivas C, Pollnau M. Organic solid-state integrated amplifiers and lasers. Laser Photon Rev 2012; 6: 419-462.

89 Yang J, Diemeer MB, Grivas C, Sengo G, Driessen A et al. Steady-state lasing in a solid polymer. Laser Phys Lett 2010; 7: 650-656.

90 Grisvas C, Yang J, Diemeer MB, Driessen A, Pollnau M. Continuous-wave Nd-doped polymer lasers. Opt Lett 2010; 35: 1983-1985.

This work is licensed under a Creative Commons AttributionNonCommercial-ShareAlike 3.0 Unported license. To view a copy of this license, visit http://creativecommons.org/licenses/by-nc-sa/3.0 